\title{
MULTIWAVE NONLINEAR COUPLINGS IN ELASTIC STRUCTURES
}

\author{
D. A. KOVRIGUINE, G. A. MAUGIN, AND A. I. POTAPOV \\ Received 27 December 2004; Revised 13 April 2005; Accepted 4 May 2005
}

This short contribution considers the essentials of nonlinear wave properties in typical mechanical systems such as an infinite straight bar, a circular ring, and a flat plate. It is found that nonlinear resonance is experienced in all the systems exhibiting continuous and discrete spectra, respectively. Multiwave interactions and the stability of coupled modes with respect to small perturbations are discussed. The emphasis is placed on mechanical phenomena, for example, stress amplification, although some analogies with some nonlinear optical systems are also obvious. The nonlinear resonance coupling in a plate within the Kirchhoff-Love approximation is selected as a two-dimensional example exhibiting a rich range of resonant wave phenomena. This is originally examined by use of Whitham's averaged Lagrangian method. In particular, the existence of three basic resonant triads between longitudinal, shear, and bending modes is shown. Some of these necessarily enter cascade wave processes related to the instability of some mode components of the triad under small perturbations.

Copyright (c) 2006 D. A. Kovriguine et al. This is an open access article distributed under the Creative Commons Attribution License, which permits unrestricted use, distribution, and reproduction in any medium, provided the original work is properly cited.

\section{Introduction}

The main aim of the first part of this short contribution is to display the essentials of nonlinear waves properties in mechanical systems of engineering origin (structural members). Nonlinear resonance is examined in two one-dimensional examples, an infinite straight elastic bar and a thin elastic circular ring, exhibiting continuous and discrete spectra, respectively. Three-wave and four-wave interactions and the stability of coupled modes with respect perturbations are discussed, the emphasis being placed on mechanical phenomena (e.g., stress amplification), although analogies with some nonlinear optical systems are obvious.

The phenomenon of nonlinear resonance coupling classically occurs in physical systems that are governed by distinct modes of propagation; these may be of similar physical

Hindawi Publishing Corporation

Mathematical Problems in Engineering

Volume 2006, Article ID 76041, Pages 1-11

DOI 10.1155/MPE/2006/76041 
nature, for example, various mechanical modes, or they may be of totally different nature-say, mechanical and magnetic or electric. In any case the two basic ingredients needed are (i) the existence of multimodes in nonlinear physical systems and (ii) the dispersion of these modes in the linearized case. Such physical situations have received the attention of applied mathematicians and wave specialists in various fields of physics and engineering science, for example, in nonlinear optics and radiophysics [16], in fluid dynamics [3], and in elastic crystals with a microstructure [13]. In the case of elastic crystals the multimodes are due to a coupling of classical elastic degrees of freedom with the kinematics of an internal structure - a rigid mechanical one such as in micropolar media and liquid crystals, a magnetic one such as in ferromagnets (coupling between phonons and magnons), and an electric one in ferroelectric bodies (electroelastic couplings).

In the present paper we focus attention on the nonlinear wave couplings in engineering elastic structures, more particularly in this contribution, in one-dimensional examples one related to an elastic infinitely long straight bar and the other to a thin closed circular ring. These two structures are chosen because they exhibit a continuous spectrum and a discrete one (due to the circular periodicity), respectively. They have, therefore, the value of paradigms. They are perfect examples of nonlinear oscillatory systems exhibiting a hierarchy of wave instabilities. The mathematical tools used are those of nonlinear science, essentially asymptotics. The related algebra often is cumbersome and will, therefore, be omitted most of the time. It can be found in lengthy original reports. We emphasize here on the mechanical consequences of the analysis. A two-dimensional example (elastic plate) is sketched out in Section 4 where the notion of cascade wave process is evoked.

\section{Nonlinear waves in a thin infinitely long bar}

In a nondimensional notation the relevant basic field equations are the following ones [6]:

$$
u_{t t}-u_{x x}=\frac{\mu}{2} \partial_{x} w_{x}^{2}, \quad w_{t t}+\alpha^{2} w_{x x x x}=\mu \partial_{x}\left(u_{x} w_{x}\right)+\frac{\mu^{2}}{2} w_{x}^{3}
$$

where $u$ is the longitudinal displacement of the middle line of the bar, $w$ is the transverse displacement, $\alpha$ is the nondimensional radius of inertia of the bar, and $\mu$ is a coupling parameter supposed to be sufficiently small for asymptotic considerations. Equations (2.1) are established under the working hypotheses of Bernoulli and Euler. Only second-order couplings between the longitudinal mode $u$ and the bending mode $w$ are kept at most. The linear analysis of (2.1) yields straightforwardly the modes as nondispersive direct and counter propagating longitudinal waves of frequency

$$
\omega_{l}= \pm k
$$

and highly dispersive bending waves of frequency

$$
\omega_{b}= \pm \alpha k^{2}
$$

The spectra are sketched in Figure 2.1. Now we consider the possible coupling between three waves selected at working points 1,2 , and 3 in this figure in a typical parallelogram 


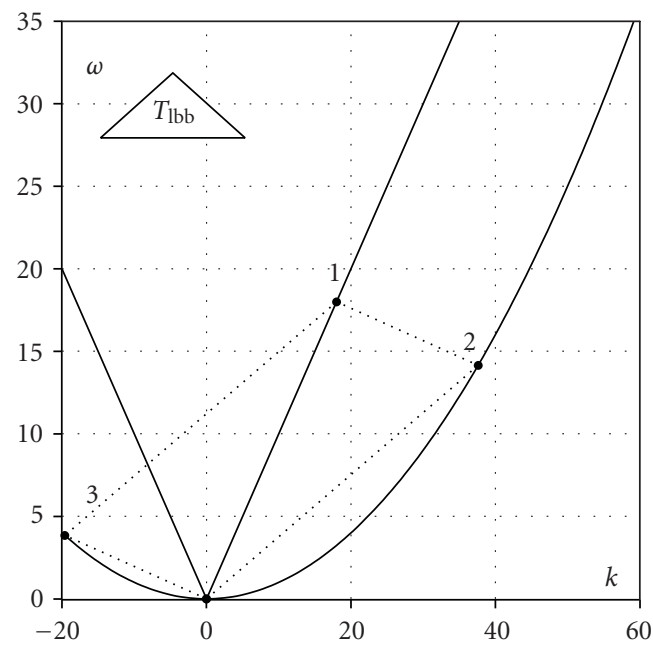

Figure 2.1. Triple-wave resonant coupling in a bar. A triad depicted as a triangle.

form such that we satisfy the so-called three-wave phase matching

$$
\omega_{3}=\omega_{1}+\omega_{2}+\Delta \omega, \quad k_{3}=k_{1}+k_{2}+\Delta k .
$$

That is, we consider the problem of modal energy exchange between a large-amplitude high-frequency longitudinal wave (point 1) coupled to two low-frequency bending waves (points 2 and 3) propagating in opposite directions. The three frequencies thus selected are said to form a resonant triad, or in a more music-like Pittagorician fashion, a resonant trio. The nonlinear resonant coupling between these modes is now examined on the basis of (2.1) at order one in the small coupling parameter $\mu$. Coupled solutions are sought in the form

$$
\begin{gathered}
u(x, t)=A_{3}(\chi, \tau) \exp i \Phi_{3}+\mu u^{(1)}(x, t)+\left(^{*}\right), \\
w(x, t)=A_{1}(\chi, \tau) \exp i \Phi_{1}+A_{2}(\chi, \tau) \exp i \Phi_{2}+\mu w^{(1)}(x, t)+\left(^{*}\right),
\end{gathered}
$$

where $\chi=\mu x, \tau=\mu t(\mu \ll 1)$, the $A_{n}$ are slowly varying amplitudes of the parts of (2.5) that are solutions satisfying the linear field equations (they are thus determined by initial and boundary conditions), and the phases $\Phi_{n}$ are such that

$$
\Phi_{n}=\omega_{n} t-k_{n} x
$$

with each couple $\left(\omega_{n}, k_{n}\right)$ satisfying the correspondingly numbered dispersion relation and altogether the phase matching conditions. The symbolism $(*)$ denotes the complex conjugate. On substituting from (2.5) into (2.1) and averaging the resulting equations 
4 Multiwave nonlinear couplings in elastic structures

over the phases, we obtain a system of three coupled hyperbolic partial differential equations for the amplitudes:

$$
\frac{\partial A_{n}}{\partial \tau}+v_{n} \frac{\partial A_{n}}{\partial \chi}=\frac{\beta}{\omega_{n}} \frac{\partial U}{\partial A_{n}^{*}},
$$

where

$$
\begin{gathered}
v_{n}=\frac{d \omega_{n}\left(k_{n}\right)}{d k}, \\
\beta=-\frac{k_{1} k_{2} k_{3}}{2}, \\
U=A_{1} A_{2} A_{3}^{*} \exp i \Delta \omega t+A_{1}^{*} A_{2}^{*} A_{3} \exp (-i \Delta \omega t)
\end{gathered}
$$

are, respectively, the group velocities of the "linear" modes, a coefficient of nonlinearity, and what may be called a cubic average potential. The Cauchy problem associated with (2.7) requires the initial conditions $A_{n}(\chi, 0)=a_{n}(\chi), n=1,2,3$.

On setting

$$
E_{n}=\omega_{n}^{2}\left|A_{n}\right|^{2}, \quad S_{n}=v_{n} E_{n}
$$

the energy and energy flux associated with each linear mode, we can establish several consequences of (2.7)-(2.8) such as the equation

$$
\frac{\partial}{\partial \tau}\left(E_{1}+E_{2}+E_{3}\right)+\frac{\partial}{\partial \chi}\left(S_{1}+S_{2}+S_{3}\right)=0
$$

clearly a law of conservation of energy between the three modes, and equations of the type

$$
\frac{\partial}{\partial \tau}\left(\frac{E_{1}}{\omega_{1}}-\frac{E_{2}}{\omega_{2}}\right)+\frac{\partial}{\partial \chi}\left(\frac{S_{1}}{\omega_{1}}-\frac{S_{2}}{\omega_{2}}\right)=0, \quad \frac{\partial}{\partial \tau}\left(\frac{E_{1}}{\omega_{1}}-\frac{E_{3}}{\omega_{3}}\right)+\frac{\partial}{\partial \chi}\left(\frac{S_{1}}{\omega_{1}}-\frac{S_{3}}{\omega_{3}}\right)=0,
$$

and similar ones by permutation. Equations (2.12), (2.13) are canonical ones. As such, they are formally identical to those obtained for three-wave mixing in nonlinear optics [16]. Direct consequences of these are the well-known Manley-Rowe relations (first integrals of (2.12)-(2.13) that characterize the energy partition between modes):

$$
\begin{gathered}
E=E_{1}+E_{2}+E_{3}=\text { const } \\
\frac{E_{1}}{\omega_{1}}-\frac{E_{2}}{\omega_{2}}=c_{1}, \quad \frac{E_{2}}{\omega_{2}}+\frac{E_{3}}{\omega_{3}}=c_{2}, \quad \frac{E_{1}}{\omega_{1}}+\frac{E_{3}}{\omega_{3}}=c_{3},
\end{gathered}
$$

where the $c_{n}$ are constants.

Remarkably, for spatially uniform processes $(\partial / \partial \chi=0),(2.7)$ yields the reduced equations

$$
\frac{d A_{n}}{d \tau}=\frac{\beta}{\omega_{n}} \frac{\partial U}{\partial A_{n}^{*}} .
$$


These are identical to the Euler equations of motion for a rigid body about a fixed point (for real-valued variables, obviously [11]). At the degree of approximation (cf. (2.5)) of the present approach, we have the following easily established results concerning the stability of modes.

(i) Longitudinal waves are unstable with respect to small low-frequency perturbations (so-called break-up instability).

(ii) Bending waves are stable-(at least) within the present first-order nonlinear approximation — with respect to small high-frequency perturbations.

(iii) The loss of stability against the high-frequency wave can lead to a dynamic stress growth caused by the resonant excitation of two low-frequency waves.

As a consequence, one must pay special attention to the initial stress level, for example, one may envisage a restriction on it so as to stay in the elastic regime. Finally, one may inquire about the temporal evolution of the considered triad. This requires exploiting a technique such as the inverse scattering method in the general case [10], or to find out much simpler analytical expressions (in terms of Jacobian elliptic functions). One may also remark that the physical system considered may exhibit triple-wave envelope solitons (a typical wave kinematical pattern) - the three amplitudes travel then together as a "complex of solitonic shapes" - in which case the two modes, say, 1 and 2 are "bright" solitons, while the third mode 3 be a "dark" one (in the optical jargon) so that energy is conserved. In this case, the amplitudes being fixed once and for all, there is no energy exchange while the triad travels inertially at constant speed.

\section{Waves traveling around a closed circular ring}

This is only briefly sketched out in order to emphasize the differences with the infinite bar of Section 2. In this case, which necessarily implies periodicity, the ring is viewed as a slice of an infinitely long thin shell and it obviously presents a discrete spectrum circumferentially.

Special attention is paid to the dynamical loss of stability against axisymmetric oscillations caused by a radially uniform impact. The nonlinearity yields nontrivial dynamical effects and we observe the existence, via modal exchanges of energy, of resonant triads between high-frequency axisymmetric oscillations and bending traveling waves (of same wave number), leading eventually to the instability of low-frequency bending waves (at the second order of approximation). The basic equations are those of thin walled shells in the geometrically nonlinear theory. Let $\varphi$ be the azimuthal angle and $R$ the radius of the ring of thickness $h$. If $v(\varphi, t)$ and $w(\varphi, t)$ are the nondimensional azimuthal displacement of the middle line and the nondimensional transverse (radial) displacement, it is convenient to introduce the following change of dependent variables:

$$
V=v_{\varphi}+w, \quad W=w_{\kappa}-v
$$

Accordingly, the equations governing $v$ and $w$ are derived from a Lagrangian formulation with Lagrangian $L$ such that

$$
L=K-\Pi
$$


with kinetic and potential energies given (in dimensional units) by

$$
K=\frac{1}{2} \rho h\left(v_{t}^{2}+w_{t}^{2}\right), \quad \Pi=\frac{1}{2} \int_{-h / 2}^{h / 2} E \varepsilon_{\varphi \varphi}^{2} d \zeta,
$$

where $E$ is Young's modulus, and the azimuthal strain $\varepsilon_{\varphi \varphi}$ is given by [5]:

$$
\varepsilon_{\varphi \varphi}=\frac{1}{R}\left(v_{\varphi}+w\right)-\frac{\zeta}{R^{2}}\left(w_{\varphi}-v\right)_{\varphi}+\frac{1}{2 R^{2}}\left(w_{\varphi}-v\right)^{2},
$$

where $\zeta \in[-h / 2, h / 2]$ is the radial distance from the middle line in a cross section of the ring. The corresponding nondimensional field equations, on account of (3.1), are deduced as

$$
\begin{gathered}
v_{t t}-V_{\varphi}+\varepsilon^{2} W_{\varphi \varphi}=\frac{\mu}{2} \partial_{\varphi}\left(w_{\varphi}^{2}\right) \\
w_{t t}+V+\varepsilon^{2} W_{\varphi \varphi \varphi}=\mu\left[\left(w_{\varphi} V\right)_{\varphi}-\frac{1}{2} w_{\varphi}^{2}\right]+\frac{\mu^{2}}{2} \partial_{\varphi}\left(w_{\varphi}^{3}\right) .
\end{gathered}
$$

Here again $\mu \ll 1$, and the relative thickness of the ring is defined by $\varepsilon=h / \sqrt{12} R$. The latter eventually provides a second small parameter.

The circular shape of the ring imposes that solutions of (3.5) satisfy the periodicity conditions

$$
v(\varphi, t)=v(\varphi+2 \pi, t), \quad w(\varphi, t)=w(\varphi+2 \pi, t) .
$$

The linear wave phases will be of the form

$$
\Phi_{k, n}=\omega_{k, n} t+n \varphi
$$

where $n$ is an integer. To each one of the angular frequencies $\omega_{k, n}$ there will correspond a normal wave. We can play with the existence of the second small parameter $\varepsilon$. In particular, we may consider the case of rings with small curvature (large radius) for which $\varepsilon \ll 1$. Then the linear coupling between modes in (3.2) with vanishing right-hand sides can be said to be weak. On discarding $\mu$-terms, (3.5) yield the following separate dispersion relations whenever we implement the inextensibility condition of the middle line of the ring $V=0$; see [4]:

$$
\omega_{1, n}= \pm \varepsilon n \frac{n^{2}-1}{\sqrt{n^{2}+1}}, \quad \omega_{2, n}= \pm \sqrt{n^{2}+1},
$$

for bending and azimuthal waves, respectively. In these conditions it is shown that the amplitudes of the azimuthal, $A_{k, n}$, and bending, $B_{k, n}$, components of the normal waves are linearly related by

$$
B_{k, n}=-i p_{k, n} A_{k, n}
$$

with coefficients $p_{k, n}(k=1,2)$, given approximately by

$$
p_{1, n}=n^{-1}, \quad p_{2, n}=-n .
$$




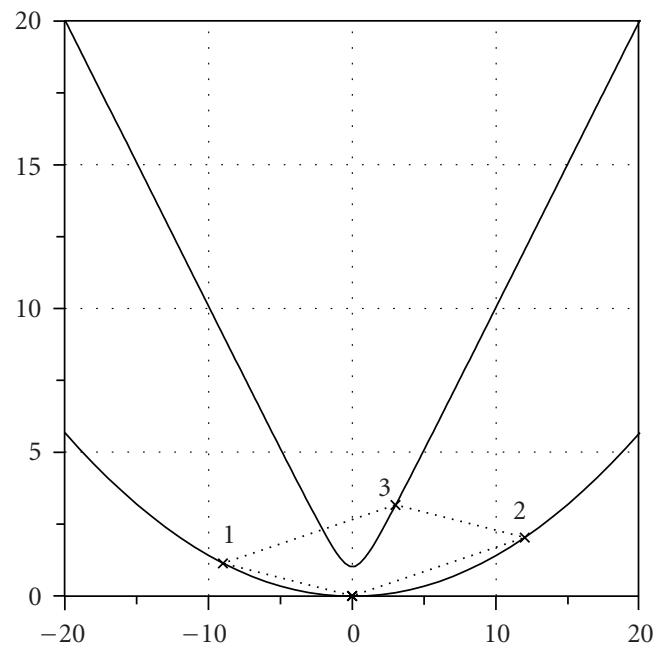

Figure 3.1. Triple-wave resonant coupling in a ring.

The linear dynamical solution (3.8)-(3.10) is usually considered as quite satisfactory. In order to respect the long-wave limit approximation, the values of $n$ should be bounded from above by some maximal wave number $n$, for example, by a characteristic wavelength that should not exceed a certain number of ring thickness (e.g., $\lambda \geq 10 h$ ). If the simplifying hypotheses applied to obtain the approximations (3.8) do not apply, then we cannot discard in (3.8) terms coming from the left-hand sides of (3.5). The linear dispersion relations then read

$$
\omega_{k, n}^{2}=\frac{1}{2}\left(n^{2}+1\right)\left(1+\varepsilon^{2} n^{2}\right) \mp \frac{1}{2}\left[\left(n^{2}+1\right)^{2}\left(1+\varepsilon^{2} n^{2}\right)^{2}-4 \varepsilon^{2} n^{2}\left(n^{2}-1\right)^{2}\right]^{1 / 2} .
$$

The proportionality coefficients $p_{k, n}$ of the amplitudes in (3.9) are now given by a more complicated frequency-dependent formula such as

$$
p_{k, n}=\frac{n\left(1+\varepsilon^{2} n^{2}\right)}{\left(1+\varepsilon^{2}\right) n^{2}-\omega_{k, n}^{2}},
$$

with the orthogonality condition $p_{1, n} p_{2, n}=-1$.

Three-wave nonlinear coupling. System (3.5) is prone to exhibiting resonance couplings inside wave triads. Exact phase matching can be realized as in Figure 3.1-here between a high-frequency azimuthal wave and two low-frequency bending waves traveling in the same direction. A particular case of this is given in Figure 3.2 describing a so-called twoto-one internal resonance between the axisymmetric oscillation and two bending waves. The algebra in the ring case is somewhat similar to that performed in Section 2 and will not be repeated. 


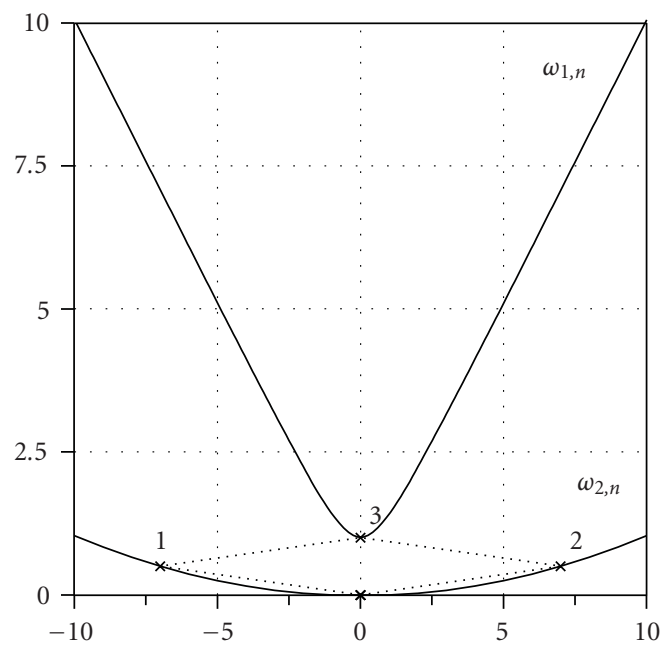

Figure 3.2. Axisymmetric phase matching.

The phase matching conditions read

$$
\omega_{1}+\omega_{2}=\omega_{3}+\Delta \omega, \quad n_{1}+n_{2}=n_{3},
$$

where $\Delta \omega$ is the detuning (equal to zero in exact matching conditions). Solutions of (3.5) are looked for in the following asymptotic form:

$$
\begin{aligned}
& v(x, t)=-i \sum_{k=1}^{3} p_{k} A_{k}(\tau) \exp i \Phi_{k}+\mu v^{(1)}(\varphi, t)+\left({ }^{*}\right), \\
& w(x, t)=\sum_{k=1}^{3} A_{k}(\tau) \exp i \Phi_{k}+\mu w^{(1)}(\varphi, t)+(*),
\end{aligned}
$$

at order $\mu$ with $\tau$ the slow time scale. Substituting from these into (3.5) and equating the terms of order $\mu$, after integration over the wave phases, we find the differential equations that govern the nonresonant corrections $v^{(1)}$ and $w^{(1)}$ as

$$
\frac{d A_{n}}{d \tau}=-i \frac{\beta}{\omega_{n} \psi_{k}^{2}} \frac{\partial U}{\partial A_{n}^{*}},
$$

where $\beta=n_{1} n_{2}\left(1-p_{1} n_{1}\right)$ is the nonlinearity coefficient, and $U$ is the average potential given by

$$
U=A_{1} A_{2} A_{3}^{*} \exp i \Delta \omega t+A_{1}^{*} A_{2}^{*} A_{3} \exp (-i \Delta \omega t) .
$$

Initial conditions associated to (3.15) read

$$
A_{n}(\chi, 0)=a_{n}(\chi), \quad n=1,2,3 .
$$


Just like in Section 2, (3.15) possesses first integrals in the form of the energy conservation and the Manley-Rowe relations (cf. [10], for details). The system being conservative (Hamiltonian), the following conclusions can be drawn in so far as stability propertiesat this order of approximation-are concerned:

(i) azimuthal high-frequency waves are unstable with respect to small perturbations (so-called break-up instability);

(ii) bending low-frequency waves are stable with respect to small perturbations;

(iii) one can study the time evolution of the amplitude envelopes [10];

(iv) the loss of stability against the high-frequency wave and the resonant excitation of two low-frequency waves is accompanied by a stress amplification phenomenon.

Notice that one can play with the value of the parameters - by adjusting them-so that matching conditions can be exactly satisfied. The critical value of $\varepsilon$ is found by solving the general expression (3.11) for fixed $n$ when we want, for instance, degenerate resonant conditions such as (this is realized in Figure 3.2)

$$
n=n_{1}=-n_{2}, \quad n_{3}=0, \quad \omega_{1}=\omega_{2}=\omega_{3} / 2 .
$$

\section{Two-dimensional example}

We finally focus our attention on the nonlinear wave couplings in an exemplary twodimensional (in space) example, provided by nonlinear waves in a thin elastic plate modeled within the Kirchhoff-Love framework. Linear modes of the longitudinal, shear, and bending types are coupled by nonlinearity. This allows one to define the interactions between the dispersion manifolds with the possibilities of group-velocity and phase-velocity matching. Weakly nonlinear waves are studied on the basis of Whitham's average Lagrangian theory $[13,14]$. This is seldom exploited in solid mechanics, notable exceptions being in $[7,15]$. This is the main originality of this contribution. For lack of space only some of the possibilities of nonlinear resonance couplings are exhibited. The study reveals that among the possible resonant triads that can be identified exhibiting phase matching and the appropriate nonlinear coupling, only three provide the building blocks of further wave constructs, namely, the $T_{\mathrm{lbb}}$-type triad (see the upper subsection) and $T_{\mathrm{sbb}^{-}}, T_{\mathrm{bsb}^{-}}$ type triads composed by shear and bending modes. Indeed, a brief study of the evolution of resonant triads shows that some of these are isolated while others have unstable components that inevitably interact with other triads (Figure 4.1). This yields the concept of cascade wave processes following along ideas of Richardson and Landau. We emphasize the necessarily sketchy nature of this paper as cumbersome formulas of repetitive form are only to be found in a long report $[10]$ and also some of our recent papers $[8,9]$. This does not exhaust the subject matter since direct numerical solutions of the true threedimensional equations would be most instructive. Other techniques such as the higherorder Bubnov-Galerkin approach and finite-element methods are promising. For these we refer the reader to recent synthesis works $[1,2,4-12,14,15,17,18]$.

The one-dimensional structural examples briefly examined in this contribution have revealed the essential properties of three-wave and four-wave resonance couplings [8]. Although the emphasis has been placed on mechanical consequences of these couplings, the analogy with nonlinear optical systems (of which the jargon is often used) is more than 


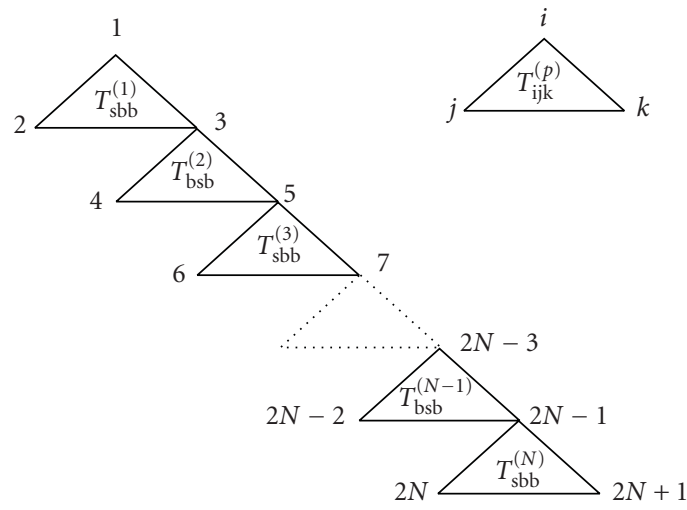

Figure 4.1. A typical triad-cell cascade in a plate. Coupling of various $T_{\mathrm{sbb}}$ - and $T_{\mathrm{bsb}}$-type triads of different scales.

obvious. This may be even more true when dealing with two-dimensional mechanical systems such as plates and shells, an example of which will be dealt within [9]. In this case the obtained results are comparable with those achieved in various physical applications, see, for instance, $[1,2,12,17,18]$. From the mathematical viewpoint this contribution should emphasize the perturbation analysis based on the idea of normal form of nonlinear PDE governing equations in a contrast with that dealing with nonlinear normal oscillatory forms $[1,2]$. This contribution has been suggested during the International Conference "Nonlinear Dynamics" held at the Kharkov Polytechnic University (Ukraine) in 2004. The subject of the paper is related to a project supported by RFBR (no. 04-0217156) and a grant from the President of the Russian Federation (no. NSh-1638.2003.8). GAM benefits from a Max Planck Award for International Cooperation (2002-2005).

\section{References}

[1] I. Andrianov, J. Awrejcewicz, and L. I. Manevitch, Asymptotical Mechanics of Thin-Walled Structures. A Handbook, Springer, Berlin, 2004.

[2] J. Awrejcewicz, I. Andrianov, and L. I. Manevitch, Asymptotic Approaches in Nonlinear Dynamics. New Trends and Applications, Springer Series in Synergetics, Springer, Berlin, 1998.

[3] A. D. D. Craig, Wave Interactions and Fluid Flows, Cambridge University Press, Cambridge, 1985.

[4] L. H. Donnell, Beams, Plates and Shells, McGraw-Hill, New York, 1979.

[5] J. H. Ginsberg, Dynamic stability of transverse axisymmetric waves in circular cylindrical shells, Transactions of the ASME. Journal of Applied Mechanics 41 (1974), 77-82.

[6] H. Kauderer, Nichtlineare Mechanik, Springer, Berlin, 1958.

[7] D. A. Kovriguine, Nonlinear resonant interactions in elastic structural members, Ph.D. thesis, Nizhny Novgorod University, Nizhny Novgorod, 1992.

[8] D. A. Kovriguine, G. A. Maugin, and A. I. Potapov, Multiwave non-linear couplings in elastic structures. I. One-dimensional examples, International Journal of Solids and Structures 39 (2002), no. 21-22, 5571-5583.

[9] _ Multiwave nonlinear couplings in elastic structures. II. Two-dimensional examples, Journal of Sound and Vibration 263 (2003), 1055-1069. 
[10] D. A. Kovriguine, A. I. Potapov, and G. A. Maugin, Multi-wave nonlinear coupling in elastic structures, Report INTAS Program 96-2370, Nizhny Novgorod, Russia and Paris, France, 2001.

[11] L. D. Landau and E. M. Lifshitz, Mechanics, 3rd ed., Pergamon, Oxford, 1976.

[12] J. Lee, Triad-angle locking in low-order models of the $2 D$ Navier-Stokes equations, Physica D. Nonlinear Phenomena 24 (1987), no. 1-3, 54-70.

[13] G. A. Maugin, Nonlinear Waves in Elastic Crystals, Oxford Mathematical Monographs, Oxford University Press, Oxford, 1999.

[14] G. A. Maugin and H. Hadouaj, Solitary surface transverse waves on an elastic substrate coated with a thin film, Physical Review B 44 (1991), no. 3, 1266-1280.

[15] G. A. Maugin, H. Hadouaj, and B. A. Malomed, Nonlinear coupling between SH surface solitons and Rayleigh modes on elastic structures, Physical Review B 45 (1992), no. 17, 9688-9694.

[16] D. F. Nelson, Electric, Optic and Acoustic Interactions in Dielectrics, John Wiley \& Sons, New York, 1979.

[17] F. Pellicano, M. Amabily, and M. P. Päidoussis, Stability of empty and fluid-filled circular cylindrical shells subjected to dynamic axial load: nonlinear dynamics of shells with fluid-structure interaction, NATO CLG grant rep., F. Pellicano, Yu. Mikhlin and I. Zolotarev eds., Institute of Thermomechanics AS CR, Prague, 2002, pp. 169-186.

[18] C. H. Riedel and C. A. Tan, Coupled, forced response of an axially moving strip with internal resonance, International Journal of Non-Linear Mechanics 37 (2002), no. 1, 101-116.

D. A. Kovriguine: Mechanical Engineering Institute, Russian Academy of Sciences,

85 Belinsky Street, 603024 Nizhny Novgorod, Russia

G. A. Maugin: Laboratoire de Modélisation en Mécanique (UMR 7607 CNRS), Université Pierre et Marie Curie, 4 Place Jussieu, 75252 Paris Cedex, France

E-mail address: gam@ccr.jussieu.fr

A. I. Potapov: Mechanical Engineering Institute, Russian Academy of Sciences, 85 Belinsky Street, 603024 Nizhny Novgorod, Russia

E-mail address: apotapov@sandy.ru 


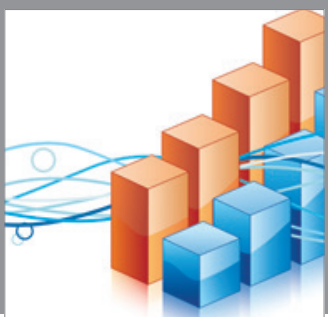

Advances in

Operations Research

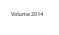

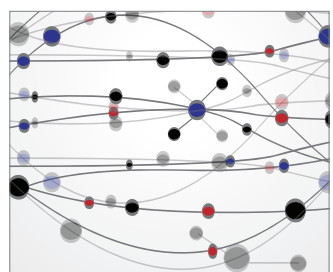

\section{The Scientific} World Journal
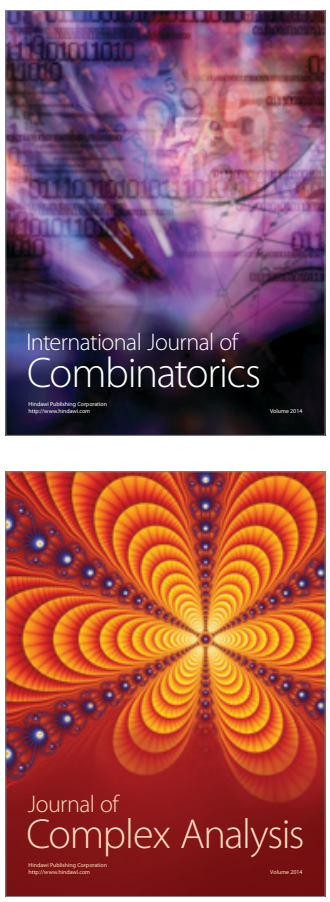

International Journal of

Mathematics and

Mathematical

Sciences
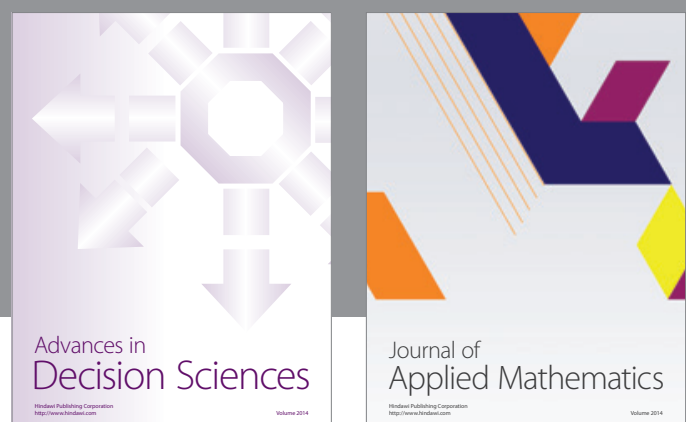

Journal of

Applied Mathematics
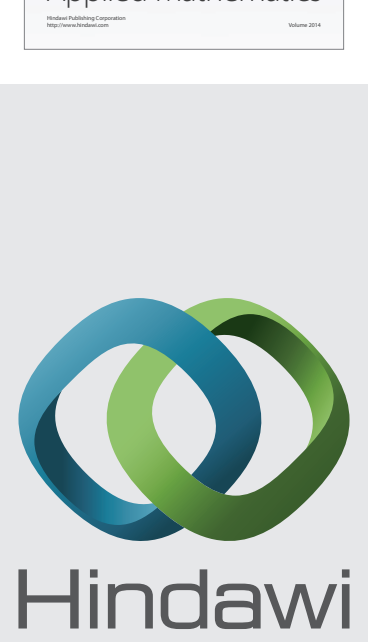

Submit your manuscripts at http://www.hindawi.com
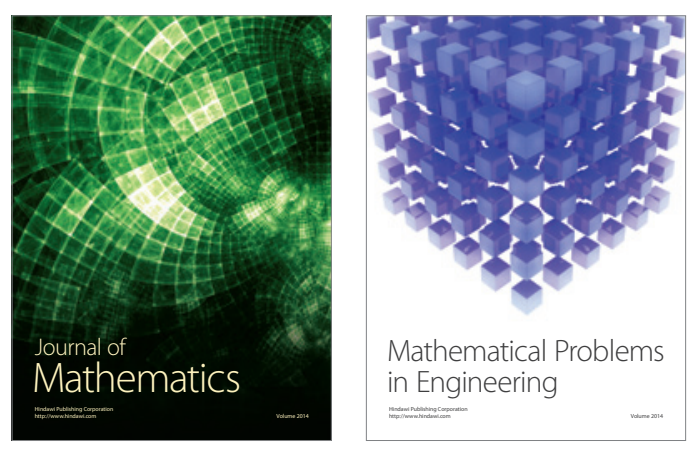

Mathematical Problems in Engineering
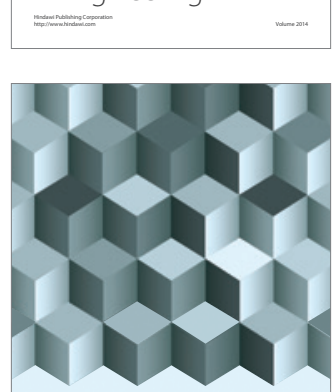

Journal of

Function Spaces
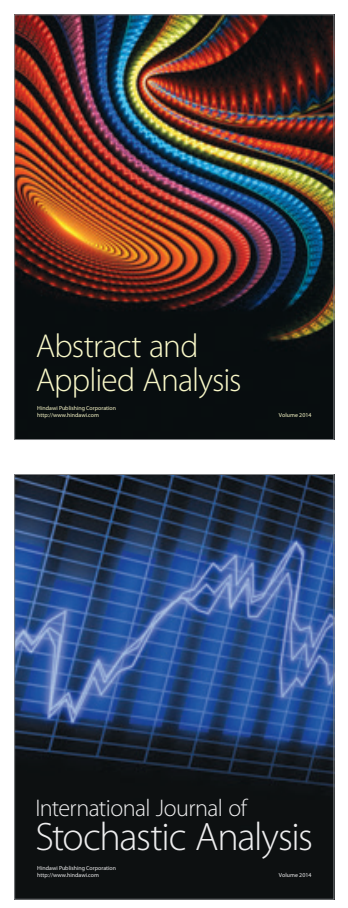

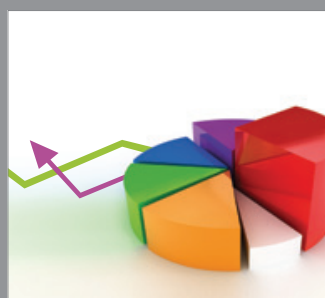

ournal of

Probability and Statistics

Promensencen
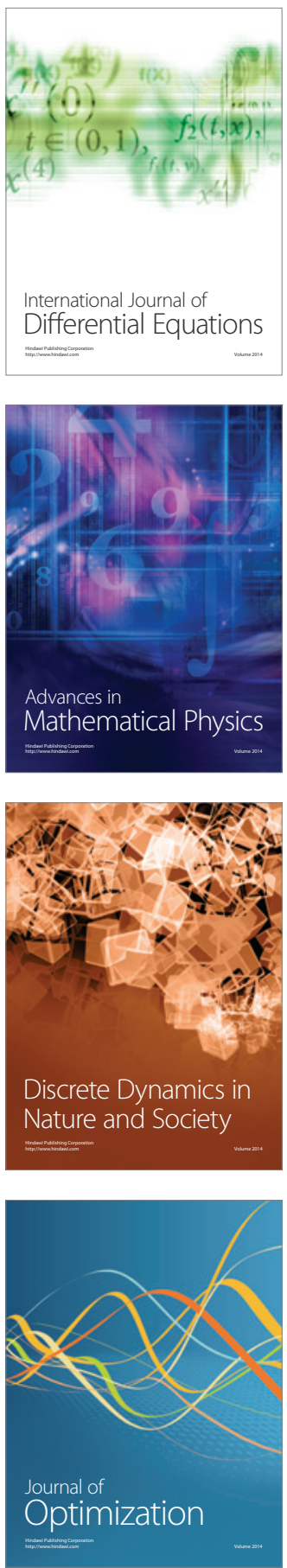